\title{
JUEGOS MUSICALES EN EL DESARROLLO DE EXPRESIÓN Y APRECIACIÓN MUSICAL DE LOS ESTUDIANTES
}

\author{
Percy Carlos Morante Gamarra ${ }^{1}$ \\ Universidad Nacional de Educación Enrique Guzmán y Valle
}

\section{RESUMEN}

El presente artículo tiene como objetivo determinar en qué medida los juegos musicales desarrollan las capacidades de expresión y apreciación musical en los estudiantes. El diseño que se ha utilizado es el cuasiexperimental. La muestra estuvo conformada por dos secciones de estudiantes del cuarto grado, donde la sección "A" fue el grupo experimental compuesta por 34 estudiantes, y la sección "C" fue el grupo control, conformado por 33 estudiantes. A ambos grupos se les aplicó la prueba de aptitud musical, posteriormente se aplicaron los juegos musicales al grupo experimental durante 5 meses en 25 sesiones de 50 minutos cada una. Se evaluó nuevamente al grupo experimental y al grupo control, observándose que el grupo experimental evidencia un avance significativo en cuanto al logro de las capacidades de expresión y apreciación musical, en comparación con el grupo control que, si bien presenta un desarrollo en relación a la evaluación inicial, ésta no es representativa.

Palabras Clave: Juego musical, expresión musical, apreciación musical, creatividad

\section{MUSICAL GAMES IN THE DEVELOPMENT OF MUSICAL EXPRESSION AND APPRECIATION OF STUDENTS}

\begin{abstract}
The objective of this article is to determine to what extent musical games develop the musical expression and appreciation abilities of students. The design that has been used is the quasi-experimental.

The sample consisted of two sections of fourth grade students, where the "A" section was the experimental group composed of 34 students, and the " $C$ " section was the control group, made up of 33 students. The musical aptitude test was applied to both groups, then the musical games were applied to the experimental group during 5 months in 25 sessions of 50 minutes each. The experimental group and the control group were evaluated again, observing that the experimental group shows a significant advance in the achievement of musical expression and appreciation abilities, in comparison with the control group that, although it presents a development in relation to the initial evaluation, this is not representative.
\end{abstract}

Key Words: Musical game, musical expression, musical appreciation, creativity

Recibido 04-03-2019. Aceptado 22-06-2019

DOI: https://doi.org/10.26752/revistaparadigmassh.v1i1.456

\section{Introducción}

La escuela de hoy, en concordancia con los avances y tendencias actuales, se ha visto en la necesidad de replantear sus currículos, con el propósito de adaptarlos a los nuevos escenarios que surgen como producto de la capacidad inventiva e innovadora del hombre.

\footnotetext{
${ }^{1}$ Doctor en Ciencias de la Educación, Doctor en Gestión Universitaria, Maestro con mención en Docencia Universitaria e Investigación Educativa, expositor en congresos a nivel nacional e internacional, autor de publicaciones relacionadas a la creatividad y arte, fue condecorado con el Premio Nacional de educación HORACIO 2004, Palmas Magisteriales 2017, Medalla de Honor 2019 del colegio de Profesores del Perú. Docente Principal, con más de 20 años de experiencia en el nivel universitario, correo: repcycarlos@hotmail.com
} 
Actualmente en el Perú, se ha generalizado el énfasis en el desarrollo de las habilidades comunicativas y matemáticas en los diferentes niveles educativos. Esta decisión, ha limitado y restringido el aprendizaje de otras áreas, las que erróneamente son percibidas como complementarias. Dentro de ellas, el área de arte es aquella que se ha visto afectada en cuanto al tiempo y contenido. Desde una perspectiva reduccionista, la escuela la ha relegado a cumplir un rol de esparcimiento y recreación en el mejor de los casos.

Comprender la importancia que tiene el arte en la vida de toda persona, es reconocer su real dimensión e influencia para una sólida formación integral; la música, actividad comprendida dentro del área artística, no es ajena a la vida del niño. Está presente desde sus primeras etapas y forma parte de sus experiencias sonoras tempranas. Son muchos los beneficios que le brinda al niño, por ello la escuela debe responder a estas necesidades e intereses, organizando adecuadamente las actividades musicales acorde a los niveles y ciclos de estudios.

Para la pedagogía contemporánea la educación musical no es complemento de una educación integral como es parte de ella misma. Los nuevos movimientos le dan una importancia decisiva en el desenvolvimiento intelectual, espiritual, emocional y social del individuo, Lo que sucede es que en cada etapa de la vida va quedando un sedimento diluido en la personalidad y permanecen en ella los efectos producidos por las experiencias; subsisten pues el producto de ese cultivo en forma de conductas diversas. En todo esto las vivencias artísticas son determinantes (Pantigoso, 1994).
En la formación integral del niño, el arte le da la posibilidad de descubrir y buscar soluciones y respuestas diferentes. Los niños deben ir encontrando en sus búsquedas personales diferentes formas de comunicar su mundo personal. Hacer uso del arte les permitirá concretar su capacidad de acción, su desarrollo perceptivo y afectivo, su progresiva toma de conciencia social y su capacidad creadora. Para Lowenfeld y Lambert (2008), probablemente el momento decisivo para la estimulación del pensamiento creativo sea cuando el niño comienza la escolaridad regulada. (...) Los niños crean con ayuda del conocimiento que pueden tener en ese momento. El acto mismo de crear puede proporcionar ideas nuevas y conocimiento nuevo para acciones posteriores. (p.97).

El arte juega un rol en el desarrollo de la integración y cohesión social y en la construcción de la identidad etaria y cultural propia. Es una herramienta para acercarse a los problemas colectivos y para la búsqueda de soluciones (Jiménez, Aguirre, Pimentel, 2009).

En relación a la música se sabe que desde las primeras edades los niños disfrutan con la música y lo demuestran tranquilizándose o atendiendo como si realmente la entendieran. En principio es el timbre el parámetro del sonido que más atrae su atención, más tarde irán descubriendo la altura, la intensidad y la duración.

A través de las actividades musicales el niño aprende placenteramente a coordinar mejor sus conocimientos, a manejarse en el espacio, a controlar el tono muscular y relajarse, aprende a escuchar, desarrolla su capacidad de comunicación y su creatividad, puede expresar sus emociones y aprende a crear 
y trabajar en grupo, a la vez que desarrolla confianza en sí mismo.

La Música, como recurso pedagógico, enriquece la formación integral del niño, no solo por su aspecto formativo sino también por su aporte en el sano desarrollo del individuo, de su personalidad. Entre las ventajas más significativas de la música está el desarrollo del aspecto intelectual, socio afectivo, psicomotor, de crecimiento personal y formación de hábitos, en lo cual se evidencia su aplicación como herramienta para la formación en valores en la educación y por consiguiente, un elemento transformador de la sociedad: "La educación musical brindará a los niños la posibilidad de abrirse a la escucha, a la exploración sonora, a la ejecución individual y grupal, al canto, al reconocimiento de la propia voz". (Mairet y Malvicini, p16, 2014).

La música cumple una función muy importante en el desarrollo socio-efectivo del niño al enseñar a diferenciar roles y definir responsabilidades, lo capacita para una mayor y mejor participación en el aula, en la relación con los compañeros y con el docente al compartir o interactuar con ellos a través de juegos y actividades musicales (canto y ejecución instrumental), dirigidas fundamentalmente a ejercitar destrezas.

Los niños y niñas experimentan emociones y espontaneidad, así como también el crecimiento de sus sentimientos estéticos. La música produce placer y satisfacción, despierta la observación y aceptación de todo cuanto lo rodea, facilita la integración grupal al compartir cantando y tocando los instrumentos con los compañeros, lo que refuerza, a su vez, la noción de trabajo cooperativo y otros indicadores de buena convivencia, como el respeto a la diversidad y el amor a sus semejantes. Según
Waisburd y Edmenger (2008). Sostienen que las habilidades musicales y los aprendizajes significativos a través de la música resultan sólo si hay disposición y apertura de vivir la experiencia, es el estado que conduce al desarrollo de la percepción y sensibilización del ser humano, fundamentos básicos para el crecimiento y el desarrollo de la persona. (p.15).

Los niños y niñas se sensibilizan ante el arte y la naturaleza, descubren un nuevo medio de expresión y comunicación, fortalecen su autoestima, conocen y expresan sus capacidades, demuestra su perseverancia en el alcance de metas; se motiva a superar dificultades cuando, al participar en producciones artísticas, se esfuerza en aplicar correctamente los elementos básicos de la música.

Todo aprendizaje natural es integrado (no lineal) porque procede desde lo global a lo particular, y activo porque aborda de manera directa el contacto con la realidad, sin esquivar ni demorar el conocimiento de la misma. Los modelos pedagógico-musicales actuales deberían centrarse en la práctica, integrar los diferentes estilos musicales, incluir las nuevas tecnologías, reflejar los gustos musicales, estudiar las diferentes formas de autoaprendizaje, interesarse en las pedagogías musicales abiertas, etcétera. (Hemsy de Gainza, 2002).

La educación musical en el aula tiene sentido cuando es una acción placentera, esto es, cuando se concibe la música como objeto de conocimiento que se escucha, que se toca, que se comprende, que se inventa y que se disfruta. Ubicar esta disciplina en la oferta optativa del currículo supone ignorar que su puesta en práctica efectiva suscita emociones 
inigualables, pone en juego elaboraciones abstractas de alto nivel, basadas en percepciones multidimensionales, permite interpretaciones disfrutables para todos y brinda oportunidades para la producción original. La necesidad de considerar a la educación musical como un área esencial en el currículo escolar, es una afirmación que durante décadas ha generado la atención de los especialistas en música y pedagogía. Como señala Zorrilllo, (2009): "Ha sido desde hace mucho tiempo y de muchas maneras discutida y por todos aceptada la importancia de estos dos aspectos: juego y música, para el crecimiento armónico del niño y es porque van de la mano con el desarrollo y precisamente por esto son esenciales para un equilibrado proceso de aprendizaje y socialización. (p.15).

Por lo tanto, aquella creencia, principalmente mantenida por quienes hacían de la enseñanza musical una labor esencialmente técnica y alambicada de corcheas, fusas y semifusas, va perdiendo consistencia ante ésta otra que defiende el carácter natural y espontáneo de lo musical identificando ritmo musical con ritmo vital y relacionando la práctica del canto y de la danza con las manifestaciones más expresivas y emotivas del sujeto.

Es de urgente la necesidad, que nuestros docentes asuman una actitud más favorable frente a la enseñanza de la música, de allí que el presente estudio tenga como propósito constituirse en una herramienta didáctica de ayuda permanente, para el docente que trabajo en el nivel primario y le permita a sus estudiantes ser parte de esta aventura valiosísima, como es la práctica del arte musical en la escuela.

El presente trabajo de investigación propone un conjunto de juegos musicales rigurosamente seleccionados, buscando que propicien en los estudiantes el desarrollo de las capacidades de expresión y apreciación musical, necesarias para fortalecer su formación integral. Así mismo constituye una herramienta didáctica que puede ser utilizada por los docentes del nivel primario, para la cual es necesario contar una base teórica elemental.

\section{Descripción de la propuesta:}

La propuesta consta de 25 juegos musicales seleccionados y adaptados para atender las necesidades de expresión y apreciación musical de los estudiantes de cuarto grado de primaria. Estas actividades lúdicas se caracterizan por incorporar aspectos básicos que plantea el currículo de educación primaria, los mismos que se desarrollan considerando la etapa de vida en que se encuentran los niños, además de responder a sus intereses musicales en concordancia con su contexto, Los juegos musicales no poseen un carácter competitivo, buscan integrar y promover en el estudiante su participación libre, espontánea para que disfruten de la música, a la vez que se van apropiando de aspectos básicos que le sirvan de soporte para posteriores aprendizajes musicales, que gradualmente se integrarán en su formación.

Asimismo, para la planificación de los juegos se tiene en cuenta el ambiente, el mobiliario, el tiempo y los recursos con que cuenta en la comunidad, los mismos que se adecúan y adaptan según el propósito de cada actividad. Para un desarrollo adecuado de la propuesta, los juegos musicales se agrupan según el aspecto que se quiere estimular 


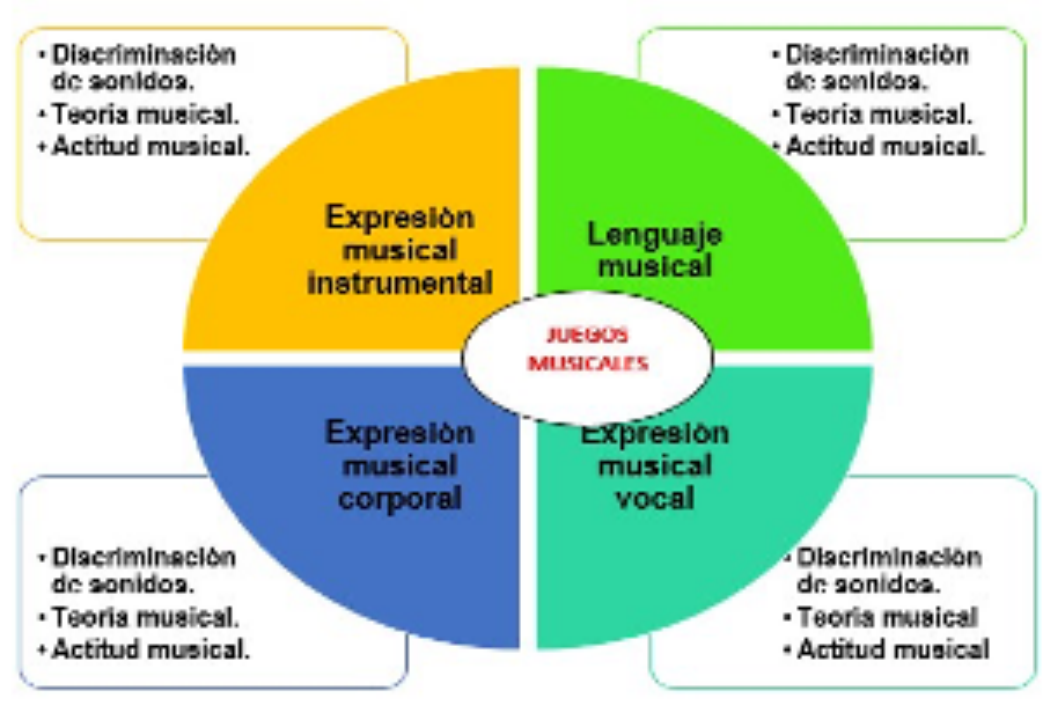

\section{Metodología de los juegos musicales}

Para la aplicación de los juegos musicales se tuvo en cuenta la siguiente planificación:

\section{Antes de juego:}

- Considerar la edad de los niños y el grado de madurez al seleccionar el juego.

- Los juegos propuestos son flexibles, por lo que se deja a la iniciativa del docente su adaptación, adecuación y modificación.

- El maestro cumple el rol de animador; sin embargo, puede en el transcurso de la actividad intercambiar roles con los estudiantes y asumir el papel de participante según el juego lo requiera. Storns. (2005).

- Se buscará enfatizar en el empleo de música propia de la región en un momento inicial, para luego incorporar melodías clásicas del repertorio nacional y mundial.

- Los juegos tendrán una duración estimada, teniendo en cuenta el grado de interés y participación de los niños.

- Los juegos se organizarán considerando el ambiente, los recursos con que se cuenta y priorizando el trabajo en equipo.

\section{Durante la actividad}

- El docente al iniciar el taller deberá explicar adecuadamente como se realiza el juego, para ello es necesario que se realice una demostración cuando el juego lo requiera. Morante. (2013)

- Considerar que se presentan situaciones donde los niños tienen temor al inicio, por lo tanto, se debe generar un clima de confianza, estimulando su participación gradual.

- Por su naturaleza, los juegos no tienen un carácter competitivo, su propósito es despertar en el niño su interés por la música, por ello es importante que el docente durante el desarrollo del juego, acompañe a los niños y atienda adecuadamente a las inquietudes que se presenten.

- Después de la actividad

- Promover la participación y el diálogo acerca de los logros obtenidos y las dificultades si fuera el caso.

- Incentivar la práctica de la escucha y la relajación.

- Complementar el juego con una actividad que se relacione, enfatizando en la práctica continua. 


\section{Programación de los juegos.}

Considerando los horarios que se destinan para el área de arte y asimismo adecuando los juegos a los momentos de las clases se ha flexibilizado su aplicación, buscando optimizar el tiempo propuesto.

\begin{tabular}{|c|c|c|c|c|}
\hline \multirow[t]{2}{*}{ No } & \multirow[t]{2}{*}{ JUEGO MUSICAL } & \multicolumn{2}{|c|}{ ASPECTO } & \multirow[t]{2}{*}{ DURACIÓN } \\
\hline & & $\begin{array}{l}\text { EXPRESIÓN } \\
\text { MUSICAL } \\
\end{array}$ & APRECIACIÓN MUSICAL & \\
\hline 01 & DESCUBRIENDO EL PULSO & Lenguaje Musical & Simbología Musical & $90 \mathrm{~min}$ \\
\hline 02 & JUEGO RITMICO & $\begin{array}{l}\text { Expresión musical } \\
\text { corporal }\end{array}$ & Simbología Musical & $45 \mathrm{~min}$ \\
\hline 03 & SONIDOS DE PAPEL & $\begin{array}{l}\text { Expresión musical } \\
\text { instrumental }\end{array}$ & Actitud Musical & $45 \mathrm{~min}$ \\
\hline 04 & CANCIONES EN EL AIRE & $\begin{array}{l}\text { Expresión musical } \\
\text { corporal }\end{array}$ & $\begin{array}{l}\text { Discriminación De } \\
\text { sonidos }\end{array}$ & $45 \mathrm{~min}$ \\
\hline 05 & SONIDO Y SILENCIO & Lenguaje Musical & $\begin{array}{l}\text { Discriminación De } \\
\text { sonidos }\end{array}$ & $45 \mathrm{~min}$ \\
\hline 06 & BUSCANDO A MIS AMIGOS & $\begin{array}{l}\text { Expresión musical } \\
\text { vocal }\end{array}$ & $\begin{array}{l}\text { Discriminación De } \\
\text { sonidos }\end{array}$ & $45 \mathrm{~min}$ \\
\hline 07 & ESPEJOS MUSICALES & Lenguaje Musical & $\begin{array}{l}\text { Discriminación De } \\
\text { sonidos }\end{array}$ & $45 \mathrm{~min}$ \\
\hline 08 & LAS ESTATUAS & $\begin{array}{l}\text { Expresión musical } \\
\text { corporal }\end{array}$ & $\begin{array}{l}\text { Discriminación De } \\
\text { sonidos }\end{array}$ & $45 \mathrm{~min}$ \\
\hline 09 & QUE INSTRUMENTO FALTA & $\begin{array}{l}\text { Expresión musical } \\
\text { instrumental }\end{array}$ & $\begin{array}{l}\text { Discriminación De } \\
\text { sonidos }\end{array}$ & $45 \mathrm{~min}$ \\
\hline 10 & DICTADO RITMICO & Lenguaje Musical & Simbología Musical & $90 \mathrm{~min}$ \\
\hline 11 & AROS MUSICALES & $\begin{array}{l}\text { Expresión musical } \\
\text { instrumental }\end{array}$ & $\begin{array}{l}\text { Discriminación De } \\
\text { sonidos }\end{array}$ & $45 \mathrm{~min}$ \\
\hline 12 & TITERECANTO & $\begin{array}{l}\text { Expresión musical } \\
\text { corporal }\end{array}$ & $\begin{array}{l}\text { Discriminación De } \\
\text { sonidos }\end{array}$ & $90 \mathrm{~min}$ \\
\hline 13 & PINTACANTOS & $\begin{array}{l}\text { Expresión musical } \\
\text { corporal }\end{array}$ & $\begin{array}{l}\text { Discriminación De } \\
\text { sonidos }\end{array}$ & $90 \mathrm{~min}$ \\
\hline 14 & PAJARITOS CANTORES & $\begin{array}{l}\text { Expresión musical } \\
\text { vocal }\end{array}$ & $\begin{array}{l}\text { Discriminación De } \\
\text { sonidos }\end{array}$ & $90 \mathrm{~min}$ \\
\hline 15 & DIRECTOR DE ORQUESTA & $\begin{array}{l}\text { Expresión musical } \\
\text { corporal }\end{array}$ & $\begin{array}{l}\text { Discriminación De } \\
\text { sonidos }\end{array}$ & $45 \mathrm{~min}$ \\
\hline 16 & $\begin{array}{l}\text { DESCUBRIENDO } \\
\text { INSTRUMENTOS }\end{array}$ & Lenguaje musical & $\begin{array}{l}\text { Discriminación De } \\
\text { sonidos }\end{array}$ & $45 \mathrm{~min}$ \\
\hline 17 & CUENTO CON SONIDOS & $\begin{array}{l}\text { Expresión musical } \\
\text { instrumental }\end{array}$ & $\begin{array}{l}\text { Discriminación De } \\
\text { sonidos }\end{array}$ & $90 \mathrm{~min}$ \\
\hline 18 & REEMPLAZANDO PALABRAS & $\begin{array}{l}\text { Expresión musical } \\
\text { vocal }\end{array}$ & Actitud Musical & $45 \mathrm{~min}$ \\
\hline 19 & $\begin{array}{l}\text { CREAMOS INTRUMENTOS DE } \\
\text { PERCUSIÓN }\end{array}$ & $\begin{array}{l}\text { Expresión musical } \\
\text { instrumental }\end{array}$ & Actitud Musical & $90 \mathrm{~min}$ \\
\hline 20 & DRAMATICANTOS & $\begin{array}{l}\text { Expresión musical } \\
\text { vocal }\end{array}$ & Actitud Musical & $90 \mathrm{~min}$ \\
\hline 21 & CREANDO CANCIONES & $\begin{array}{l}\text { Expresión musical } \\
\text { vocal }\end{array}$ & Actitud Musical & $90 \mathrm{~min}$ \\
\hline 22 & $\begin{array}{l}\text { CUADNO LAS CANCIONES } \\
\text { CRECEN }\end{array}$ & $\begin{array}{l}\text { Expresión musical } \\
\text { vocal }\end{array}$ & Actitud Musical & $45 \mathrm{~min}$ \\
\hline 23 & CANCIONES EN DESRODEN & $\begin{array}{l}\text { Expresión musical } \\
\text { vocal }\end{array}$ & Actitud Musical & $90 \mathrm{~min}$ \\
\hline 24 & CANON & $\begin{array}{l}\text { Expresión musical } \\
\text { vocal }\end{array}$ & Actitud Musical & $90 \mathrm{~min}$ \\
\hline 25 & GRAVE Y AGUDO & Lenguaje musical & Simbología Musical & $90 \mathrm{~min}$ \\
\hline
\end{tabular}




\section{Métodología}

La investigación utilizada en este trabajo, es de enfoque cuantitativo; aunque, igualmente. Es importante decir que se hizo uso del método cuantitativo, al considerar que los datos del estudio han sido analizados y cuantificados, utilizándose como técnicas para la recolección de datos, el cuestionario para medir las aptitudes de apreciación y expresión musical.

\section{Participantes:}

La población está conformada por 141 estudiantes del 4to grado de primaria de la I.E. $N^{\circ}$ 10104, distribuidos en cuatro aulas A, B, C y D. La muestra del estudio estuvo constituida por 02 secciones. (Sección A, 34 estudiantes, grupo experimental y sección C, 33 estudiantes, grupo control).

\section{Materiales:}

Técnicas e instrumentos de recolección de datos: Análisis de documentos, Observación, el cuestionario para evaluar las capacidades musicales, Prueba de pre test, Prueba de post test.

\section{Resultados}

Tabla 1.

Prueba de Kolmogorov-Smirnov para una muestra

\section{Pruebas de normalidad}

\begin{tabular}{lcccccc} 
& \multicolumn{3}{c}{ Kolmogorov-Smirnova } & \multicolumn{3}{c}{ Shapiro-Wilk } \\
\cline { 2 - 7 } & Estadístico & gl & Sig. & Estadístico & gl & Sig. \\
\cline { 2 - 7 } GCPRE &, 263 & 33 &, 049 &, 847 & 33 &, 054 \\
GCPOS &, 254 & 33 &, 067 &, 833 & 33 &, 066 \\
GEPRE &, 153 & 34 &, $200^{*}$ &, 969 & 34 &, 882 \\
GEPOS &, 254 & 34 &, 067 &, 833 & 34 &, 066 \\
\hline
\end{tabular}

\section{Tabla 2.}

Cuadro comparativo de resultados de evaluación de la capacidad de apreciación musical

\begin{tabular}{lcc} 
& GE pre test & GE pos test \\
\hline Media & 9.39393939 & 13.9411765 \\
Varianza (conocida) & 3.83 & 1.99 \\
Observaciones & 34 & 34 \\
Diferencia hipotética de las medias & 4.5 & \\
$z$ & -21.824154 & \\
$P(Z<=z)$ una cola & 0 & \\
Valor crítico de $z$ (una cola) & 1.64485363 & \\
Valor crítico de $z$ (dos colas) & 0 & \\
Valor crítico de $z$ (dos colas) & 1.95996398 & \\
\hline
\end{tabular}


Actualmente parece oportuno plantear la educación artística a través del juego como forma de incentivar el interés de nuestros alumnos, ya que los juegos proporcionan aprendizajes que pueden ser transferidos a conocimientos de distinta índole que pueden aplicarse en distintas técnicas y diferentes campos artísticos. Esta conclusión concuerda con los resultados de nuestra investigación al analizar el avanceenel logro de las capacidades de expresión y apreciación musical en base en los resultados de las pruebas realizadas, tanto para el grupo experimental como al grupo control, se puede afirmar que fue positivo. Esto teniendo en cuenta, que, en promedio, los puntajes de las pruebas finales fueron superiores a los de las pruebas iniciales.

Asimismo, Carbajo (2009) en su trabajo de investigación, buscó caracterizar al maestro de música, a partir de la reflexión que ellos mismos realizan acerca de su práctica educativa. Consideró la importancia del empleo de metodologías que se basen en el juego como una forma de lograr que los estudiantes adquieran las capacidades que la educación musical plantea. En la presente investigación A partir del estadístico de prueba z se realizó la comparación de los valores medios de las puntuaciones de cambio de los grupos experimental y control, respecto al pre test y al pos test. Los resultados de esta prueba arrojan como conclusión que la diferencia es significativa y no sólo es debida a la aleatoriedad de las mediciones.

Deloanterior, se acepta la hipótesis alternativa y por ende se puede asegurar que los juegos musicales desarrollan las capacidades de expresión y apreciación musical en los estudiantes del 4to grado de la I.E. No 10104. Lambayeque-2014 de forma positiva, es decir, desarrollando sus capacidades artísticas.
En el estudio realizado por Cruces (2009). Se señala que la música beneficia también el desarrollo socioemocional del niño al propiciar la aceptación de sí mismo con sus posibilidades y límites, además que mediante la experiencia músico-artística se cultivan y desarrollan también los sentidos del niño, promoviéndose así el desarrollo perceptivo. Esta conclusión justifica la necesidad de utilizar la música en la escuela como un recurso que contribuye a fortalecer la formación de quiénes son partícipes de ella. Por ello en nuestro estudio realizado, los juegos musicales aplicados al grupo experimental, muestra mejor resultados que la aplicada al grupo de control ya que: la media del grupo experimental arroja 14,11puntuaciones en cambio el grupo de control arroja 11,8 puntuaciones, la diferencia es de 2 puntuaciones, la cual es significativa.

Los resultados de la presente investigación demuestran la importancia y los efectos favorables que tienen las prácticas musicales en la escuela. En concordancia con Longueira (2011) que investigó la problemática de la educación musical y concluyó señalando La música como ámbito de educación general, es decir, como ámbito que forma parte de la educación común de los escolares y desarrolla el sentido estético y de lo artístico por medio de contenidos y formas de expresión musicales.

\section{Conclusiones}

A partir de los resultados obtenidos, se concluye al $95 \%$ de nivel de confianza que se ha determinado que los juegos musicales desarrollan las capacidades de expresión y apreciación musical en los estudiantes del 4to grado de la I.E. No 10104. Lambayeque-2014. Esto se demostró con los resultados del contraste de hipótesis (calculado=-17,7 
cae en la zona de aceptación de la hipótesis alterna) y figuras pertinentes.

Esto se demostró con los resultados del contraste de hipótesis ((calculado=-30,91 Lo que cae en la zona de aceptación de la hipótesis alterna) y figuras pertinentes.

Teniendo como base la segunda hipótesis específica de la investigación, se concluye igualmente como en las anteriores hipótesis que el 95\% de nivel confianza que los juegos musicales desarrollan las capacidades de apreciación musical en los estudiantes del 4to grado de la I.E. No 10104. Lambayeque-2014 Esto se demostró con los resultados del contraste de hipótesis (Z-calculado=-21,82 cae en la zona de aceptación de la hipótesis alterna) y figuras pertinentes.

\section{Referencias Bibliográficas}

1. Carbajo, C. (2009). "El perfil profesional del docente de música de educación primaria: autopercepción de competencias profesionales y la práctica de aula". (Tesis doctoral). Universidad de Murcia. España.

2. Cruces, M. (2009). "Implicaciones de la expresión musical para el desarrollo de la creatividad en educación infantil. Málaga 2009". (Tesis Doctoral) Universidad de Málaga. España.

3. Hemsy de Gainza, V (2002). Pedagogía musical. (3 reimp.) Argentina: Lumen

4. Jiménez, L., Aguirre, I.y Pimentel, L. (2009). Educación artística, cultura y ciudadanía. España: Anzos. SL.

5. Longueira, S. (2011). "Educación musical: un problema emergente de intervención educativa. Indicadores pedagógicos para el desarrollo de competencias en educación musical. España 2011". (Tesis Doctoral). Universidad de Santiago de Compostela. España.

6. Lowenfeld, V. y Lambert, W. (2008). Desarrollo de la capacidad intelectual y creativa. España: Ed. Síntesis

7. Mairet,S. y Malvicini,K.(2014).Didáctica de la música en el nivel inicial. Argentina: Ed. Bonum.

8. Morante, P. (2013). Cantijugando. Actividades musicales para desarrollar la creatividad en los niños. Ed. Graphicomp: Perú.

9. Pantigoso, M. (1994) Educación Por el Arte. Lima.
10. Storns G. (2005). 101 juegos musicales (3era ed.). España: GRAO.

11. Waisburd, G.y Edmenger, E. (2008). El poder de la música en el aprendizaje. México: Ed. Trillas.

12. Zorrillo, A. (2009). Juego musical y aprendizaje. España: Magisterio. 